Review Paper http://ajol.info/index.php/ijbcs http://indexmedicus.afro.who.int

\title{
Gestion intégrée des principaux ravageurs du cotonnier au Sénégal et en Afrique occidentale
}

\author{
Djibril BADIANE ${ }^{1 *}$, Momar Talla GUEYE ${ }^{2}$, Emile Victor COLY ${ }^{3}$ et Ousmane FAYE ${ }^{4}$ \\ ${ }^{I}$ Institut Sénégalais de Recherches Agricoles, Centre de Recherches Zootechniques BP 53 Kolda, Sénégal. \\ ${ }^{2}$ Institut de Technologie Alimentaire, Hann-Dakar, BP 2765, Sénégal. \\ ${ }^{3}$ Institut Sénégalais de Recherches Agricoles, Centre pour le Développement de l'Horticulture, \\ BP 3120 Bel-Air Dakar, Sénégal. \\ ${ }^{4}$ Département de Biologie Animale, Faculté des Sciences de Dakar, UCAD, BP 5005, Dakar, Sénégal. \\ *Auteur correspondant ; E-mail :djibril.badiane@isra.sn, djibybadiane@gmail.com
}

\section{RESUME}

Les pertes de récolte dues aux insectes ravageurs, en culture cotonnière, restent importantes au Sénégal et en Afrique occidentale. Les solutions proposées pour combattre ces insectes nuisibles sont axées sur la lutte chimique. Toutefois, compte tenu des effets néfastes de l'utilisation des pesticides (apparition de souches résistantes, pollution de l'environnement, intoxications) la recherche d'alternatives s'impose. Les principales espèces de ravageurs rencontrées, en particulier Helicoverpa armigera (Hübner), insecte redoutable sur cotonnier, peuvent faire l'objet d'une lutte à l'aide de méthodes alternatives utilisant des produits biologiques et la sélection d'espèces résistantes aux attaques du ou des ravageur(s). Les différentes méthodes de protection phytosanitaire du cotonnier pratiquées en alternative ou combinées avec les pesticides ainsi que divers autres aspects de la problématique de gestion des insectes ravageurs du cotonnier sont passés en revue.

(C) 2015 International Formulae Group. All rights reserved.

Mots clés: Cotonnier, ravageurs, lutte, pesticides, Sénégal, Afrique Occidentale.

\section{INTRODUCTION}

Le coton occupe une place centrale dans l'économie de l'ensemble des pays producteurs en Afrique. D'après une étude de l'Union Economique et Monétaire Ouest Africain (UEMOA) réalisée en 2010, le coton constitue la principale source de revenus pour 15 à 20 millions de personnes et représente $60 \%$ des recettes d'exportation. Le coton africain représentait environ $6 \%$ des exportations mondiales en 2010 et près de $3,5 \%$ de la superficie cotonnière dans le monde (FAO, 2011). Il est un facteur important de lutte contre la pauvreté.

Toutefois, la filière cotonnière est confrontée à des difficultés telles que la baisse de fertilité des sols, la pression parasitaire et le coût élevé des intrants, qui affectent la production et impactent l'économie.

Le cotonnier est attaqué par de nombreux ravageurs dont les principaux sont les chenilles des capsules Helicoverpa armigera (Hübner) (Lepidoptera Noctuidae), Diparopsis watersi (Rothchild) (Lepidoptera - Noctuidae), Earias spp. 
(Lepidoptera - Noctuidae), les pucerons Aphis gossypii (Glover) (Homoptera - Aphididae) et les chenilles défoliatrices dont Haritalodes derogata (Fabricius) (Lepidoptera, Pyralidae) (Brévault, 2010). La pression des insectes ravageurs s'exerce sur tous les stades de développement de la plante (Badiane, 1995). La protection phytosanitaire contre ces insectes nuisibles est une des composantes majeures pour une production de coton en quantité et en qualité. Elle est basée sur la lutte chimique avec l'utilisation de pesticides de synthèse. Cette méthode de lutte a été considérée comme nécessaire et incontournable (Matthews et Tunstall, 1994). Dans des conditions optimales, le développement de stratégies de protection phytosanitaire, essentiellement basé sur l'utilisation des insecticides, a permis pendant des décennies un contrôle satisfaisant de l'ensemble des ravageurs du cotonnier. Cependant, la lutte chimique a montré ses limites avec la perte d'efficacité de bon nombre de molécules insecticides, suite à l'apparition de populations d'insectes résistants (Brévault, 2010). C'est le cas de Helicoverpa armigera (ravageur le plus redoutable) vis-à-vis des pyréthrinoïdes (PRPICA, 2013). Aussi, l'utilisation de pesticides de synthèse occasionne des intoxications des utilisateurs, une pollution de l'environnement et des désordres écologiques (Guèye, 2011). Par ailleurs, Ferron et al. (2006) indiquent qu'un rapport de l'ICAC (International Cotton Advisory Committee), paru en 1998, avait montré que la protection phytosanitaire représentait à elle seule 25 à $45 \%$ des coûts totaux de production de la culture cotonnière. La mise en œuvre depuis 1998 de nouveaux programmes de gestion de la résistance aux pyréthrinoïdes chez $H$. armigera a amené les producteurs à utiliser des produits alternatifs beaucoup plus onéreux (PR-PICA, 2013). Ces contraintes de la lutte chimique posent des problèmes de durabilité des systèmes de culture. Il s'avère donc nécessaire de promouvoir des stratégies de gestion intégrée pour assurer la durabilité de la lutte contre les ravageurs en vue de contribuer à l'amélioration de la productivité et de la qualité du coton tout en préservant l'environnement (PR-PICA, 2013).

La gestion intégrée des ravageurs du cotonnier repose sur: i) le respect d'une entomofaune naturelle épargnée du déséquilibre écologique; ii) l'exploitation de caractères de résistance du cotonnier (naturels ou introduits) pour limiter le développement de certains ravageurs tout en préservant l'environnement; iii) des pratiques culturales qui renforcent la qualité des plantes; iv) une utilisation raisonnée des pesticides chimiques. Les différents aspects de la gestion intégrée des ravageurs sont passés en revues dans le présent document.

\section{LES INSECTES DANS LE SYSTEME DE CULTURE DU COTONNIER}

L'attaque du cotonnier par les insectes représente la principale cause de destruction des récoltes de coton. Des estimations de la Conférence des Nations Unies pour le Commerce et le Développement (CNUCED) en 2003 indiquent que les pertes dues aux ravageurs représentent environ $15 \%$ de la production annuelle mondiale. Selon Oerke et Dehne (2004) et Guèye (2011), les pertes de récolte dues aux bio-agresseurs sont de l'ordre de $30 \%$ en moyenne avec $13 \%$ pour les arthropodes, $10 \%$ pour les agents pathogènes et $7 \%$ pour les mauvaises herbes. En Afrique de l'Ouest, les pertes de récolte dues aux ravageurs du cotonnier sont évaluées en moyenne à $47,96 \%$ et $15,5 \%$ du potentiel de production, respectivement dans les cultures classiques et transgéniques (PR-PICA, 2013). L'entomofaune nuisible du cotonnier est riche, diversifiée et relativement cosmopolite (Smith et Cothren, 1999). Elle est composée aussi bien d'espèces monophages que sténophages, mais aussi et surtout polyphages (Ferron et al., 2006). Ces insectes attaquent de nombreuses plantes cultivées, susceptibles d'être associées au cotonnier dans divers systèmes de culture. Ils colonisent également de nombreuses plantes non cultivées, qui peuvent ainsi jouer le rôle de plantes relais, de 
zones refuges ou de corridors biologiques et influencer la dynamique spatio-temporelle de leurs populations (Vaissayre, 1995).

\section{LES PRINCIPAUX RAVAGEURS DU COTONNIER AU SENEGAL ET EN AFRIQUE OCCIDENTALE}

Les arthropodes (myriapodes, acariens, insectes) constituent un obstacle majeur à la culture cotonnière. Du semis à la récolte, le cotonnier est attaqué par divers ravageurs. Il constitue la cible d'attaque de plusieurs espèces d'insectes (Badji, 2010).

Au Sénégal, l'entomofaune nuisible du cotonnier reste dominé par une dizaine d'espèces dont l'importance et l'incidence varient sur la production cotonnière en fonction des zones et des années (Diongue, 1989; Badji, 2010). Selon Cauquil (1993), les insectes nuisibles peuvent être classés en trois grands groupes en fonction des phases de développement de la plante. Il s'agit: des ravageurs des semis et plantules composés principalement de Diplopodes; des arthropodes de la phase végétative qui sont principalement des Lépidoptères et des Homoptères phyllophages, englobant presque tous les ordres; et des insectes de la phase reproductive dominés, essentiellement, par des Lépidoptères et quelques Hétéroptères.

\section{Les ravageurs des semis et plantules}

Au semis, la graine et la jeune plantule sont attaquées par des diplopodes (mille pattes). Selon Traoré (2008), les espèces les plus nuisibles appartiennent aux genres Peridontopyge et Tibiomus. Les iules (diplopodes) détruisent la semence avant la levée en dévorant l'amande par pénétration des téguments de la graine au niveau du hile. Ils causent aussi des lésions sur la plantule favorisant ainsi la pénétration d'agents pathogènes qui peuvent entraîner la mort de la plantule (Badiane, 1995 ; Badji, 2010).

\section{Les ravageurs de la phase végétative}

Au cours de cette phase, on distingue trois groupes de ravageurs qui sont :
- les espèces défoliatrices ou phyllophages : Spodoptera littoralis (Boisduval) (Lepidoptera, Noctuidae), Haritalodes derogata (Fabricius) (Lepidoptera, Pyralidae) et Anomis flava (Fabricius) (Lepidoptera, Noctuidae) ;

- les piqueurs-suceurs : Aphis gossypii

(Glover) (Homoptera, Aphididae), Bemisia tabaci (Gennadius) (Homoptera, Aleyrodidae) et Jacobiella fascialis (Jacobi) (Homoptera, Ciccadellidae) ;

- et les acariens : Polyphagotarsonemus latus (Banks) (Acarina, Tarsonemidae) et Tetranychus urticae (Koch) (Acarina, Tetranychidae).

La gravité des dégâts des insectes de la phase végétative réside dans la précocité de leur apparition. Ces insectes provoquent une destruction des chloroplastes bloquant ainsi l'élaboration de l'essentiel des substances organiques nécessaires à la plante (Appert et Deuse, 1988 in Badiane 1995). Dans le cas d'une attaque sévère, les chenilles dévorent les feuilles en ne laissant que les nervures principales (Badji, 2010).

\section{Les ravageurs de la phase reproductive}

Helicoverpa armigera (Hübner) (Lepidoptera, Noctuidae), insecte polyphage, est de loin le ravageur le plus important sur les capsules du cotonnier. Les autres espèces sont: Diparopsis watersi (Rothchild) (Lepidoptera, Noctuidae) qui est une espèce monophage, inféodée au cotonnier et Earias spp, représentée au Sénégal par Earias insulana (Boisduval) et Earias biplaga (Walker) (Lepidoptera, Noctuidae). La punaise rouge Dysdercus volkeri (Schmidt) (Heteroptera, Pyrrhocoridae) cause aussi d'importants dégâts sur les capsules du cotonnier (Badji, 2010).

Les insectes de la phase de reproduction sont les principaux responsables des pertes de production observées sur le cotonnier. En effet, en l'absence d'une protection contre les insectes ravageurs, les pertes de production peuvent varier entre 25 et $70 \%$. Plus de deux tiers de ces pertes sont dues aux chenilles de $H$. armigera, Earias spp 
et D. watersi. Selon les estimations, 2 à 3 chenilles de $H$. armigera par plante peuvent endommager tous les fruits en 15 jours (Brévault et al., 2002).

\section{La Noctuelle Helicoverpa armigera (Hübner)}

Helicoverpa armigera (Hübner) est le ravageur le plus régulier et le plus dangereux de la culture cotonnière. C'est une espèce très cosmopolite avec une très grande aire de répartition car présente dans toutes les zones de culture cotonnière à l'exception de l'Amérique (Tiemtoré, 2007). L'insecte a une forte fécondité et un cycle de reproduction très court. Le cycle biologique est de 42 jours à 22 ${ }^{\circ} \mathrm{C}$, ce qui permet 5 à 6 générations par an (Vaissayre, 1995). Une étude faite par Matthews et Tunstall (1994) indique que le cycle biologique de l'insecte est d'une durée de 28 à 30 jours sous une température de 25 ${ }^{\circ} \mathrm{C}$. Une femelle pond en moyenne 1000 œufs préférentiellement à la tombée de la nuit au stade de la floraison. L'éclosion des œufs se produit entre 2 et 4 jours après la ponte. La chenille, stade nuisible de Helicoverpa armigera, se développe au bout de 14 à 24 jours. Entre le $17^{\text {ème }}$ et le $21^{\text {ème }}$ jour de son développement, elle quitte la plante et donne une chrysalide (Brévault et al., 2002). La chrysalide peut éventuellement entrer en diapause lorsque les conditions sont défavorables (Badiane 1995).

Les chenilles sont activement mobiles et voraces. Elles pénètrent à l'intérieur des boutons floraux, des fleurs et des capsules pour s'alimenter et vident le contenu de ces organes florifères et fructifères. Les jeunes organes attaqués tombent après dessèchement. La floraison est retardée ou ralentie suivant les possibilités de compensation de la plante (Vaissayre, 1995). Les dégâts sur les fleurs occasionnent leur chute précoce ou une malformation des capsules dont la destruction est souvent complétée par des bactéries et des champignons secondaires. L'incidence de $H$. armigera est d'autant plus grave que chaque chenille attaque successivement plusieurs organes sur le même plant. Deux à trois chenilles suffisent pour annuler toute la fructification d'une plante au bout de deux semaines (Bruinsma, 1987). Les chenilles se nourrissent également sur les tiges (Brévault, 2010). L'impact économique du ravageur croît avec la succession des générations. Selon Brévault (2010), les attaques sont d'autant plus fortes que les pluies sont espacées.

$H$. armigera est un ravageur polyphage ; il s'attaque à d'autres plantes en plus du cotonnier notamment les cultures maraîchères comme la tomate. En dehors du cotonnier et de la tomate, $H$. armigera commet aussi des dégâts sur le maïs, le sorgho, le tabac, le poivron, le haricot, la pomme de terre, le gombo, le chou, la laitue, etc (Cauquil, 1993, Badji, 2010 ; Brévault, 2010).

La voie chimique est privilégiée pour lutter contre $H$. armigera. La détection précoce de la présence de l'insecte conditionne l'efficacité des interventions chimiques (Kébé, 1999). Des pulvérisations rapprochées aux pyréthrinoïdes s'avèrent nécessaires pour protéger les nouvelles pousses surtout dans les zones de prédilection des pontes (Badji 2010). Cependant, une certaine perte d'efficacité de ces molécules de pyréthrinoïdes est observée dans plusieurs régions du monde (Buès et Boudinhon, 2003). Cette résistance rend l'intoxication des chenilles âgées quasi impossible par les doses usuelles. L'utilisation de matières actives alternatives aux pyréthrinoïdes, lors des applications insecticides permet de lutter efficacement contre les infestations de $H$. armigera (Bal et Sagna, 2003).

Le phénomène de résistance, en plus de compromettre l'efficacité de la lutte, peut avoir des répercussions préoccupantes non seulement sur les plans économique et sanitaire, mais également écologique, par l'accroissement des doses d'insecticides utilisées. Les solutions à mettre en place pour lutter contre la résistance auraient conduit à un triplement du coût des pesticides vulgarisés 
en zones de culture cotonnière d'Afrique (Bruinsma, 1987).

Le coton $B t$, une des alternatives dans la gestion de la résistance, a la réputation d'avoir la capacité de faire face aux attaques d'Helicoverpa armigera. En effet, la recherche a réussi à développer des gènes tels que les gènes Cry $1 \mathrm{~A}(\mathrm{~b})$ et Cry $1 \mathrm{~A}(\mathrm{c})$ qui arrivent de manière partielle à contrôler la nuisibilité de la chenille (Enda diapol, 2009).

\section{LES MOYENS DE LUTTE CONTRE LES RAVAGEURS DU COTONNIER}

Pour réduire l'incidence économique des insectes ravageurs sur la production de coton, la protection phytosanitaire intègre actuellement trois modalités de lutte contre les insectes : les techniques culturales, la résistance variétale et la lutte chimique (Bruno et al., 2000). Dans un souci de répondre à des préoccupations de rentabilité économique, de santé humaine et de sécurité environnementale, la protection phytosanitaire s'est donnée pour objectif un recours minimal, aux pesticides de synthèse, par la mise en œuvre d'une approche de gestion intégrée des nuisibles (Prudent et al, 2006). Les techniques de lutte énumérées ci-dessous sont celles recommandées par la recherche et adoptées par les producteurs de coton.

\section{La lutte chimique}

En Afrique de l'Ouest, la lutte chimique reste le principal moyen de lutte contre les ravageurs du cotonnier. La première étape importante dans la protection du cotonnier est axée sur le traitement de la semence afin d'améliorer la levée et de l'état sanitaire des plantules (Traoré, 2008). La semence est ainsi désinfectée avec des associations «fongicides de contact et insecticides (systémique ou de contact) » pour faire face aux champignons microscopiques, aux ravageurs du sol (iules), ainsi qu'aux ravageurs des jeunes plants (jassides, pucerons) (Badiane, 1995). Les programmes de traitements chimiques foliaires actuellement appliqués sont basés à la fois sur un calendrier préétabli et sur des interventions sur seuil. Le Tableau 1 indique les différents principes actifs testés et recommandés au Sénégal contre les principaux nuisibles du cotonnier.

Les différents types de programmes de traitements chimiques foliaires ci-dessous sont en vigueur en Afrique de l'Ouest.

\section{Le programme de traitement calendaire}

Le programme de traitement sur calendrier permet d'assurer la protection des cotonniers durant la période allant du début de la floraison jusqu'à la maturation de la majorité des capsules formées. Le programme calendaire intègre le niveau de technicité des producteurs qui est souvent bas avec des difficultés de reconnaissance des ravageurs, mais, aussi la gestion des stocks de produits différents.

En Afrique de l'Ouest, depuis sa conception, le principe de la protection de la culture du coton sur calendrier a connu deux variantes. Il s'agit du programme standard et des programmes fenêtres.

\section{- Le programme standard}

Avec ce programme dit classique, les traitements démarraient dès l'apparition des premières fleurs, soit 45 à 50 jours après la levée. En général, la cadence recommandée entre les traitements était de 14 jours. Les producteurs réalisaient 5 à 6 traitements foliaires uniquement avec un produit binaire (pyréthrinoïde + organophosphoré) ou parfois ternaire (une pyréthrinoïde + deux organophosphorés). Cependant, l'utilisation prolongée et répétée des pyréthrinoïdes a provoqué l'apparition de résistance de certains ravageurs, notamment Helicoverpa armigera. Ceci a conduit à la révision du programme de traitements calendaire au profit de fenêtres (Traoré, 2008).

\section{- Les programmes fenêtres}

Les programmes fenêtres sont basés sur le principe des interventions sur calendrier à 14 ou 10 jours d'intervalle. Le démarrage des traitements est effectué 30-35 jours après la levée (période d'apparition des boutons floraux). La grande nouveauté est la création 
des «Fenêtres » d'intervention qui consistent à une utilisation limitée de molécules chimiques alternatives aux pyréthrinoïdes. Ainsi, sont apparus les programmes 2 fenêtres et 3 fenêtres (Traoré, 2008).

- Le programme à 2 fenêtres : selon le principe de ce programme, les traitements ayant lieu avant le 25 août forment la première fenêtre tandis que la seconde fenêtre est constituée par les autres traitements qui se déroulent à partir du 26 août jusqu'au $115^{\text {ème }}$ jour après la levée.

- Le programme à 3 fenêtres : si les traitements ayant lieu avant le 25 août forment la première fenêtre, ceux ayant lieu entre le 26 août et le 30 septembre donnent la seconde fenêtre et les traitements à partir du 01 octobre jusqu'au $115^{\text {ème }}$ jour après la levée constituent la troisième fenêtre.

Le choix des produits à appliquer s'opère avec beaucoup de discernement. En première fenêtre, pour les deux types de programmes-fenêtre, les traitements sont toujours réalisés avec un produit n'appartenant pas à la famille des pyréthrinoïdes, afin de réduire l'utilisation des molécules de cette famille contre laquelle $H$. armigera a développé de la résistance. Les molécules alternatives aux pyréthrinoïdes sont : le profenofos, l'indoxacarb, le spinosad, l'association flubendiamide-spirotétramate entre autres (Traoré, 2008). En seconde fenêtre, les traitements sont réalisés avec des produits binaires contenant un pyréthrinoïde en association avec des molécules d'autres familles. Dans le cas du programme 3 fenêtres, on peut intervenir avec des produits acaricides au niveau de la deuxième fenêtre puis des produits aphicides et/ou aleurodicides en troisième fenêtre.

Les produits acaricides tels que cyperméthrine/profenofos,

deltaméthrine/triazophos

et

aphicides/aleurodicides comme lambdacyhalothrine/acétamiprid, alphaméthrine/imidacloprid sont souvent utilisés. Dans le programme à 3 fenêtres, on peut également intervenir avec des molécules alternatives aux pyréthrinoïdes au niveau de la troisième fenêtre (Figure 1). Ce programme est actuellement vulgarisé au Sénégal pour la lutte contre les ravageurs du cotonnier.

Cette nouvelle stratégie a non seulement permis de freiner l'aggravation de la résistance de Helicoverpa aux pyréthrinoïdes, mais aussi une prise de conscience pour éviter l'apparition du même phénomène chez d'autres ravageurs de la culture cotonnière.

\section{Le programme de traitement sur seuils}

Les programmes d'interventions sur seuils consistent à effectuer les traitements après la détermination des niveaux d'infestation à partir desquels il s'avère nécessaire d'intervenir pour sauver la production. Leur expansion est encore lente pour plusieurs raisons liées à l'insuffisance de la connaissance des ravageurs qui constituent, sans aucun doute, l'élément déterminant pour la réussite de ce programme.

La conception de cette stratégie de protection doit tenir compte de la diversité des ravageurs du cotonnier. Les expertises ont été prioritairement axées sur l'évaluation de l'importance des dégâts dus aux insectes ravageurs à partir des résultats de recherche provenant d'autres régions du monde. Cette évaluation permet de prendre la décision de déclencher les traitements afin de contrôler les infestations (Traoré, 2008).

L'évaluation des niveaux d'infestation des populations d'insectes requiert les prélèvements d'échantillons permettent d'évaluer les populations de ravageurs et/ou leurs dégâts dans le champ de coton et de prendre la décision d'intervenir (Brévault 2010). Le principe de base commande d'effectuer un bon examen systématique des carpophages, phyllophages et piqueurssuceurs sur les plants échantillonnés. Les traitements sont faits généralement avec des produits spécifiques en cas d'atteinte des seuils d'intervention, ou avec des produits binaires quand la nécessité s'impose. Le choix des produits et la gestion de leurs stocks ne 
sont pas choses très aisées pour les producteurs.

\section{LA RESISTANCE DES RAVAGEURS AUX INSECTICIDES ET LA NOUVELLE STRATEGIE DE GESTION DE LA RESISTANCE}

La résistance des ravageurs en particulier de la noctuelle $H$. armigera aux pyréthrinoïdes a été particulièrement étudiée en Australie et en Asie où une résistance poussée est observée (Buès et Boudinhon, 2003). Elle a également été détectée en Afrique de l'Ouest. Les pyréthrinoïdes avaient été adoptés dans les années 80 en raison de leur efficacité à faible dose sur plusieurs ravageurs et de leur faible toxicité sur les mammifères (Héma, 2004). Le phénomène de résistance a montré la nécessité d'un sursaut. C'est ainsi que face au danger, les pays cotonniers d'Afrique de l'Ouest ont conjugué leurs efforts dans le cadre du Projet régional pour la gestion et la prévention de la résistance de Helicoverpa armigera en Afrique de l'Ouest (PR-PRAO). La résistance d'une population d'insectes donnée à un insecticide représente toute une évolution, déterminée génétiquement, de sa tolérance à cet insecticide, en réponse à une pression de sélection (Bruinsma, 1987).

La stratégie de gestion de la résistance en culture cotonnière mise en place au Sénégal et en Afrique de l'Ouest recommande: i) la suppression des pyréthrinoïdes lors des traitements ayant lieu avant le 25 août, pour limiter la sélection d'individus résistants chez la première génération, ii) les premiers traitements avec des insecticides alternatifs qui ne présentent pas de résistance croisée positive, iii) les traitements suivants avec des mélanges à base d'organophosphorés synergisants pour accroître l'efficacité des pyréthrinoïdes (Martin, 2003).

Les travaux de recherche ont montré, que l'endosulfan qui s'est révélé très efficace contre les chenilles de $H$. armigera a été choisi à la place des pyréthrinoïdes, pour lutter contre les insectes du cotonnier en début de campagne (Mbaye, 2008). Le PR-PRAO a recommandé d'utiliser l'endosulfan pour les deux premiers traitements et les associations de pyréthrinoïdes et d'organophosphorés qui augmentent l'efficacité de ces deux types d'insecticides aux traitements suivants. Les pyréthrinoïdes doivent donc obligatoirement être utilisés au stade fructifère car ils sont les seuls efficaces contre d'autres chenilles de la capsule telles que Earias spp et Diparopsis watersi entre autres.

A cause de ses impacts notoires sur la santé et l'environnement, le Comité Sahélien des Pesticides (CSP) a interdit l'utilisation de l'endosulfan dans ses Etats membres (Mbaye, 2008). Le Sénégal a ainsi interdit l'utilisation de l'endosulfan depuis 2008. De nouvelles molécules beaucoup moins toxiques telles le profénofos, l'indoxacarb et le spinosad, ont été testées avec succès et sont actuellement utilisées en substitut à l'endosulfan (Mbaye, 2008).

L'utilisation excessive de pesticides pour lutter contre les organismes nuisibles mine l'équilibre naturel de l'écosystème agricole: elle perturbe les populations de parasitoïdes et de prédateurs et provoque ainsi des infestations de ravageurs secondaires. Elle expose également les agriculteurs à de graves risques pour leur santé et à des retombées négatives pour l'environnement et, parfois, pour les rendements des cultures. Il arrive souvent que moins d'un pour cent des pesticides appliqués atteigne effectivement les organismes nuisibles visés, le reste contaminant l'air, les sols et les eaux (FAO, 2011). Il s'avère donc nécessaire de trouver des alternatives à la lutte chimique.

\section{LES ALTERNATIVES A LA LUTTE CHIMIQUE}

Les techniques culturales

Les caractères variétaux de résistance sont utilisés de façon systématique pour lutter contre certaines maladies, comme la bactériose et la fusariose. Une pilosité suffisamment élevée des variétés cultivées 
empêche l'installation et la prolifération des jassides (Traoré, 2008).

Le labour permet de détruire les billons et d'exposer les larves diapausantes et les chrysalides de certaines espèces et les insectes terricoles au soleil et aux prédateurs.

Le semis précoce et synchronisé tend à diminuer le temps de présence des infestations sur la culture. Un semis précoce, effectué au mois de mai, permet à la culture d'échapper partiellement aux infestations des ravageurs, en particulier $H$. armigera (Cadou, 1982). Selon Traoré (2008), le semis précoce permet d'éviter que la période la plus sensible pour le cotonnier ne tombe dans la deuxième génération de $H$. armigera qui provoque des dommages très importants à cette phase (Figure 2). Le semis précoce permet de minimiser les possibilités de migration et de réduire les pertes réalisées sur les semis tardifs.

La densité de semis peut avoir une influence sur le comportement de certains ravageurs. Les écartements préconisés au Sénégal sont $80 \mathrm{~cm} \times 30 \mathrm{~cm}$, ce qui donne une densité de 41.666 poquets/ha.

Les récoltes rapides après l'ouverture des capsules permettent de diminuer le degré de collage de la fibre.

L'apport optimum d'engrais augmente la vigueur de la plante et par conséquent sa capacité à supporter des dégâts ou compenser les organes perdus.

Le désherbage élimine non seulement la compétition entre la culture et les mauvaises herbes mais aussi les plantes hôtes alternatives susceptibles d'héberger des ravageurs, en particulier des hémiptères (punaises et jassides), ce qui réduit les populations de ces insectes (Bruno et al., 2000). Au Sénégal, au minimum deux sarclages suivis d'un buttage sont préconisés en dehors de l'herbicidage effectué juste après le semis avant la levée des cotonniers.
La rotation des cultures incluant des espèces de plantes non hôtes des ravageurs associés au cotonnier est essentielle pour briser le cycle de développement des insectes nuisibles. La rotation des cultures et l'assolement empêchent aussi l'émergence des populations de ravageurs, de maladies et de mauvaises herbes. En outre, le nombre de ravageurs est limité par les insectes utiles et les oiseaux qui trouvent leur habitat dans les cultures de rotation ou d'assolement (Ouédraogo et al., 2008).

La destruction des tiges après récolte permet de diminuer la densité des populations des ravageurs soit par leur destruction par le feu, soit par manque de nourriture qui les pousse à la migration. Au Sénégal, la destruction des résidus de récolte se fait avec souvent un retard car le producteur est confronté au problème de récolte des vivriers.

L'Association de cultures : les cultures les plus communément associées au coton sont le sorgho, le maïs et le niébé. Le gombo et le tournesol, cultivés avec le coton, sont utilisés comme plantes pièges (Ouédraogo et al., 2008).

\section{Les mesures préventives}

Les pièges peuvent contribuer à réduire les populations de certains ravageurs, particulièrement les papillons. Lorsqu'ils sont utilisés assez tôt, ils peuvent empêcher la massification des ravageurs (Ouédraogo et al., 2008).

Le suivi des ravageurs permet de déterminer le degré d'infestation des ravageurs et quand il est nécessaire de prendre des mesures de lutte directe.

\section{La promotion des ennemis naturels}

Les ennemis naturels permettent de maintenir les attaques de ravageurs à des niveaux raisonnables. La préservation des habitats naturels des insectes utiles et des oiseaux permettent d'augmenter les populations d'ennemis naturels du cotonnier 
dans le champ (Ouédraogo et al., 2008). Les diverses cultures situées à proximité du coton, comme celles fourragères, peuvent être une source d'ennemis naturels des nuisibles.

\section{La résistance variétale}

Les caractères variétaux de résistance sont utilisés de façon systématique pour lutter contre certaines maladies, comme la bactériose et la fusariose ; une pilosité suffisamment élevée des variétés cultivées empêche l'installation et la prolifération des jassides (Bruno et al., 2000). La sélection concerne aussi des variétés possédant des glandes à gossypol pour lutter contre les attaques des altises. D'autres variétés résistantes ont été testées en Afrique de l'Ouest, mais non pas été diffusées (PR-PICA, 2013). Ce sont les variétés à feuilles «OKRA» pour réduire le feuillage et les variétés à bractées frego contre $H$. armigera (PR-PICA, 2013).
Au Sénégal, les variétés cultivées sont issues de la recherche. Les semences sont mises à la disposition des producteurs. L'adoption de la lutte génétique actuelle par les producteurs est passive.

\section{La lutte biologique}

La lutte biologique utilise des organismes vivants ou des bactéries qui affectent les ravageurs. La plupart des produits de lutte biologique ne sont efficaces que contre un ravageur spécifique.

Des tests réalisés au Sénégal ont donné des résultats satisfaisants avec Bt (Bacillus thuringensis) - un microbe efficace contre les larves mangeuses de feuilles, VPN (virus polyhédrose nucléaire) qui s'attaque à la chenille américaine du cotonnier et l'élimine ; d'entomopathogènes, particulièrement le Baculovirus de Mamestra brassicae sur les chenilles de $H$. armigera et de D. watersi.

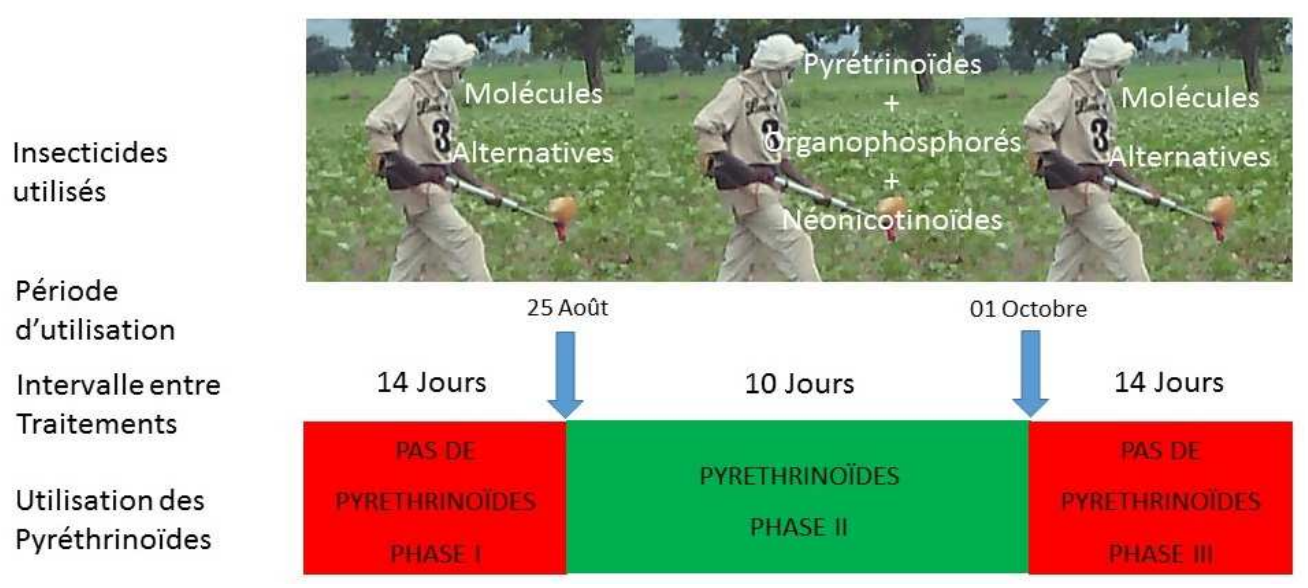

Figure 1: Programme de protection à trois «fenêtres » pour la gestion de la résistance aux pyréthrinoïdes. 


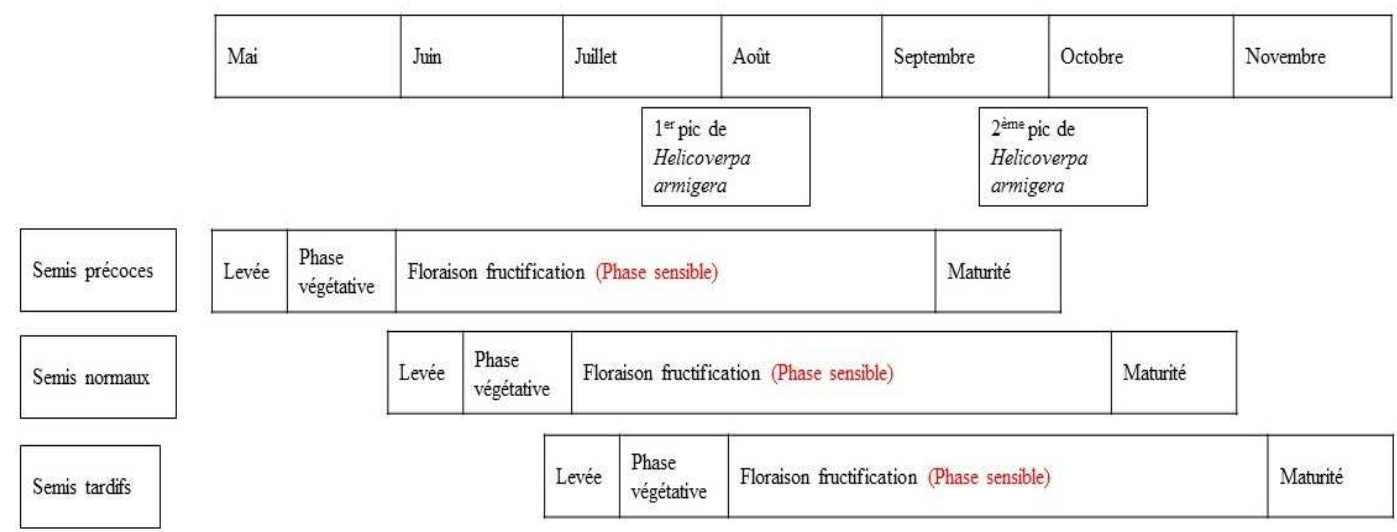

Figure 2 : Importance de la période de semis dans la protection phytosanitaire du cotonnier.

Tableau : Pesticides recommandés dans la protection du cotonnier au Sénégal.

\begin{tabular}{|c|c|c|c|}
\hline Principes actifs & Dose optimale & Nuisibles ciblés & Famille chimique \\
\hline \multicolumn{4}{|l|}{ Traitement de semences } \\
\hline Imidacloprid + Propiconazole & $(125+15 \mathrm{~g} / \mathrm{kg})$ & $\begin{array}{c}\text { Maladies, } \\
\text { insectes du sol } \\
\text { \& des plantules }\end{array}$ & $\begin{array}{l}\text { Néonicotinoïde }+ \\
\text { Triazoles }\end{array}$ \\
\hline Chlorpyriphos éthyl + Thirame & $(250+250 \mathrm{~g} / \mathrm{kg})$ & $\begin{array}{c}\text { Maladies, } \\
\text { insectes du sol } \\
\text { \& des plantules }\end{array}$ & $\begin{array}{c}\text { Organophosphoré + } \\
\text { Dithiocarbamate }\end{array}$ \\
\hline Imidacloprid + Thirame + Pencycuron & $233+107+50 \mathrm{~g} / 1$ & $\begin{array}{c}\text { Maladies, } \\
\text { insectes du sol } \\
\text { \& des plantules }\end{array}$ & $\begin{array}{c}\text { Néonicotinoïde + } \\
\text { Dithiocarbamate + } \\
\text { Phénylurée }\end{array}$ \\
\hline $\begin{array}{l}\text { Thiamethoxam + Fludioxonil + } \\
\text { Mefenoxam }\end{array}$ & $(350+25+10 \mathrm{~g} / \mathrm{l})$ & $\begin{array}{c}\text { Maladies, } \\
\text { insectes du sol } \\
\text { \& des plantules }\end{array}$ & $\begin{array}{c}\text { Néonicotinoïde + } \\
\text { Phénylpyrrole + } \\
\text { Acylamine }\end{array}$ \\
\hline Imidachlopride + TMTD & $(250+200 \mathrm{~g} / \mathrm{kg})$ & $\begin{array}{c}\text { Maladies, } \\
\text { insectes du sol } \\
\text { \& des plantules }\end{array}$ & $\begin{array}{l}\text { Néonicotinoïde + } \\
\text { Dithiocarbamate }\end{array}$ \\
\hline Imidacloprid + Thirame & $(250+100 \mathrm{~g} / \mathrm{kg})$ & $\begin{array}{c}\text { Maladies, } \\
\text { insectes du sol } \\
\text { \& des plantules }\end{array}$ & $\begin{array}{l}\text { Néonicotinoïde }+ \\
\text { Dithiocarbamate }\end{array}$ \\
\hline \multicolumn{4}{|l|}{ Traitements foliaires } \\
\hline \multicolumn{4}{|l|}{$1^{\text {ère }}$ fenêtre } \\
\hline Endosulfan* & $750 \mathrm{~g} / \mathrm{ha}$ & complexe & Cyclodiène chloré \\
\hline Profenofos & $750 \mathrm{~g} / \mathrm{ha}$ & complexe & Organophosphoré \\
\hline Spinozad & $480 \mathrm{~g} / \mathrm{ha}$ & $\begin{array}{c}\text { chenilles } \\
\text { carpophages }\end{array}$ & Spinosynes \\
\hline Chromafenozide & $50 \mathrm{~g} / \mathrm{ha}$ & $\begin{array}{l}\text { chenilles } \\
\text { carpophages }\end{array}$ & $\begin{array}{l}\text { Agonistes } \\
\text { d'hormone de muer } \\
\text { (régulateurs de }\end{array}$ \\
\hline
\end{tabular}




\begin{tabular}{|c|c|c|c|}
\hline & & & $\begin{array}{c}\text { croissance } \\
\text { d'insectes }(\mathrm{RCI}))\end{array}$ \\
\hline Indoxacarb & $150 \mathrm{~g} / \mathrm{ha}$ & $\begin{array}{c}\text { chenilles } \\
\text { carpophages }\end{array}$ & Oxadiazine \\
\hline Flubendiamide/Spirotetramate & $(20+15 \mathrm{~g} / \mathrm{ha})$ & $\begin{array}{c}\text { chenilles } \\
\text { carpophages }\end{array}$ & $\begin{array}{c}\text { Benzene- } \\
\text { dicarboxamide } \\
\text { /Acide tetronic }\end{array}$ \\
\hline Azadirachtine & & $\begin{array}{c}\text { chenilles } \\
\text { carpophages }\end{array}$ & $\begin{array}{c}\text { Botanique } \\
\text { (limonoïde) }\end{array}$ \\
\hline Lufenuron & & $\begin{array}{c}\text { chenilles } \\
\text { carpophages }\end{array}$ & Benzoylurée \\
\hline Emamectine benzoate & $19 \mathrm{~g} / \mathrm{ha}$ & $\begin{array}{c}\text { chenilles } \\
\text { carpophages }\end{array}$ & Avermectine \\
\hline Diflubenzuron & $150 \mathrm{~g} / \mathrm{ha}$ & $\begin{array}{c}\text { chenilles } \\
\text { carpophages }\end{array}$ & Benzoylurée \\
\hline Flubendiamide + Thiacloprid & $(24+24 \mathrm{~g} / \mathrm{ha})$ & $\begin{array}{l}\text { chenilles } \\
\text { carpophages }\end{array}$ & $\begin{array}{c}\text { Benzene- } \\
\text { dicarboxamide }+ \\
\text { Néonicotinoïde }\end{array}$ \\
\hline Teflubenzuron & $15 \mathrm{~g} / \mathrm{ha}$ & $\begin{array}{c}\text { chenilles } \\
\text { carpophages }\end{array}$ & Benzoylurée \\
\hline Cyantraniliprole & $40 \mathrm{~g} / \mathrm{ha}$ & $\begin{array}{c}\text { chenilles } \\
\text { carpophages }\end{array}$ & Diamide \\
\hline \multicolumn{4}{|l|}{ 2ème fenêtre } \\
\hline $\begin{array}{l}\text { Cyperméthrine }+ \text { Triazophos }+ \\
\text { Acétamiprid }\end{array}$ & $(36+150+8 \mathrm{~g} / \mathrm{ha})$ & $\begin{array}{c}\text { chenilles } \\
\text { carpophages, } \\
\text { chenilles } \\
\text { phyllophages, } \\
\text { piqueurs suceurs }\end{array}$ & $\begin{array}{c}\text { Pyréthrinoïde + } \\
\text { organophosphoré + } \\
\text { Néonicotinoïde }\end{array}$ \\
\hline Thiamethoxam + Lambdacyhalothrine & $(21+15 \mathrm{~g} / \mathrm{ha})$ & $\begin{array}{c}\text { chenilles } \\
\text { carpophages, } \\
\text { piqueurs suceurs }\end{array}$ & $\begin{array}{l}\text { Néonicotinoïde }+ \\
\text { Pyréthrinoïde }\end{array}$ \\
\hline Lambdacyhalothrine + Acétamiprid & $(15+8 \mathrm{~g} / \mathrm{ha})$ & $\begin{array}{c}\text { chenilles } \\
\text { carpophages, } \\
\text { piqueurs suceurs }\end{array}$ & $\begin{array}{l}\text { Pyréthrinoïdes + } \\
\text { Néonicotinoïde }\end{array}$ \\
\hline Lambdacyhalothrine + Profénofos & $(15+250 \mathrm{~g} / \mathrm{ha})$ & $\begin{array}{c}\text { chenilles } \\
\text { carpophages, } \\
\text { chenilles } \\
\text { phyllophages, } \\
\text { piqueurs suceurs }\end{array}$ & $\begin{array}{l}\text { Pyréthrinoïdes }+ \\
\text { Organophosphoré }\end{array}$ \\
\hline Lambdacyhalothrine + Pyriproxyfene & $(15+15 \mathrm{~g} / \mathrm{ha})$ & $\begin{array}{c}\text { chenilles } \\
\text { carpophages, } \\
\text { piqueurs suceurs }\end{array}$ & $\begin{array}{c}\text { Pyréthrinoïdes + } \\
\text { Dérivé de pyridine } \\
\text { (imitateur juvénile } \\
\text { d'hormone) }\end{array}$ \\
\hline Bétacyfluthrine + Imidacloprid & $(9+20 \mathrm{~g} / \mathrm{ha})$ & $\begin{array}{c}\text { chenilles } \\
\text { carpophages, } \\
\text { piqueurs suceurs }\end{array}$ & $\begin{array}{l}\text { Pyréthrinoïdes + } \\
\text { Néonicotinoïde }\end{array}$ \\
\hline Profénofos + Deltaméthrine & $(250+12 \mathrm{~g} / \mathrm{ha})$ & chenilles & Organophosphorés \\
\hline
\end{tabular}




\begin{tabular}{|c|c|c|c|}
\hline & & $\begin{array}{l}\text { carpophages, } \\
\text { piqueurs suceurs }\end{array}$ & + Pyréthrinoïdes \\
\hline Acétamirpide + Cyperméthrine & $(8+36 \mathrm{~g} / \mathrm{ha})$ & $\begin{array}{c}\text { chenilles } \\
\text { carpophages, } \\
\text { piqueurs suceurs }\end{array}$ & $\begin{array}{l}\text { Néonicotinoïde }+ \\
\text { Pyréthrinoïde }\end{array}$ \\
\hline $\begin{array}{l}\text { Lambdacyhalothrine }+ \text { Acétamiprid }+ \\
\text { Profénofos }\end{array}$ & $(15+8+150 \mathrm{~g} / \mathrm{ha})$ & $\begin{array}{c}\text { chenilles } \\
\text { carpophages, } \\
\text { chenilles } \\
\text { phyllophages, } \\
\text { piqueurs suceurs }\end{array}$ & $\begin{array}{l}\text { Pyréthrinoïdes + } \\
\text { Néonicotinoïde + } \\
\text { Organophosphoré }\end{array}$ \\
\hline Lambdacyhalothrine + Imidacloprid & $(15+50 \mathrm{~g} / \mathrm{ha})$ & $\begin{array}{c}\text { chenilles } \\
\text { carpophages, } \\
\text { piqueurs suceurs }\end{array}$ & $\begin{array}{l}\text { Pyréthrinoïde + } \\
\text { Néonicotinoïde }\end{array}$ \\
\hline Lambdacyhalothrine + Thiamethoxam & $(15+30 \mathrm{~g} / \mathrm{ha})$ & $\begin{array}{c}\text { chenilles } \\
\text { carpophages, } \\
\text { piqueurs suceurs }\end{array}$ & $\begin{array}{l}\text { Pyréthrinoïde + } \\
\text { Néonicotinoïde }\end{array}$ \\
\hline Bifenthrine + Acétamipride & $(30+8 \mathrm{~g} / \mathrm{ha})$ & $\begin{array}{c}\text { chenilles } \\
\text { carpophages, } \\
\text { piqueurs suceurs }\end{array}$ & $\begin{array}{l}\text { Pyréthrinoïde + } \\
\text { Néonicotinoïde }\end{array}$ \\
\hline Spinoteram + Acétamipride & $(14+16 \mathrm{~g} / \mathrm{ha})$ & $\begin{array}{c}\text { chenilles } \\
\text { carpophages, } \\
\text { piqueurs suceurs }\end{array}$ & $\begin{array}{l}\text { Spinosyne + } \\
\text { Néonicotinoïde }\end{array}$ \\
\hline Flonicamid + Bifenthrine $* *$ & $(250+15 \mathrm{~g} / \mathrm{ha})$ & $\begin{array}{c}\text { chenilles } \\
\text { carpophages, } \\
\text { piqueurs suceurs }\end{array}$ & $\begin{array}{l}\text { Néonicotinoïdes + } \\
\text { Pyréthrinoïdes }\end{array}$ \\
\hline Teflubenzuron + Alfacyperméthrine & $36 \mathrm{~g} / 1$ & $\begin{array}{c}\text { chenilles } \\
\text { carpophages, } \\
\text { piqueurs suceurs }\end{array}$ & $\begin{array}{l}\text { Benzoylurée + } \\
\text { Pyréthrinoïde }\end{array}$ \\
\hline Acéphate & $970 \mathrm{~g} / \mathrm{ha}$ & $\begin{array}{l}\text { Bemisia tabaci } \\
\text { (fin de cycle) }\end{array}$ & Organophosphoré \\
\hline
\end{tabular}

* Arrêté pour cause de toxicité

** Pas vulgarisé

\section{Conclusion}

Les différentes méthodes présentées comme alternatives aux pesticides présentent chacune des avantages, mais aussi quelques limites. La protection intégrée s'impose comme la principale stratégie holistique de protection des plantes. Elle se donne pour objectif de maintenir le bon fonctionnement des écosystèmes.

La lutte intégrée en Afrique de l'ouest contre les ravageurs du cotonnier reste fortement tributaire de la lutte chimique sans laquelle la productivité ne peut être assurée.
Toutefois, face à la mondialisation, au coût mondial trop fluctuant de la fibre de coton et aux subventions accordées aux producteurs de coton du Nord, il est nécessaire d'adopter des itinéraires techniques plus performants et une meilleure organisation des producteurs afin d'accroître la productivité au champ. De nouvelles technologies comme la production et l'utilisation de la fumure organique à l'aide d'activeurs, la protection sur seuils d'intervention et le coton génétiquement modifié peuvent bien rentrer dans ce cadre. 


\section{REFERENCES}

Badiane D. 1995. Situation parasitaire entomologique $\mathrm{du}$ cotonnier au Sénégal et méthodes de contrôle. Mémoire de titularisation, entomologie agricole, Tambacounda: Direction de Recherches sur les Cultures et Systèmes Pluviaux de l'ISRA. 81p.

Badji A. 2010. Gestion des principaux insectes ravageurs du cotonnier au Sénégal. Mémoire de fin de cycle, Ingénieur Agronome. Katibougou, Mali : Institut Polytechnique Rural de Formation et de Recherche Appliquée (IPR/IFRA), 62p.

Bal AB, Sagna SB. 2003. Les arthropodes nuisibles au cotonnier. In: Les principaux arthropodes nuisibles des céréales, des légumineuses et du cotonnier au Sahel et leur contrôle. Fascicule de cours des étudiants Ingénieurs en Protection des Végétales 2ème années, AGRHYMET Niamey, Niger. 72 p.

Brévault T, Beyo J, Nibouche S, Vaissayre M. 2002. La résistance des insectes aux insecticides Problématique et enjeux en Afrique centrale. Actes du colloque Savanes africaines : des espaces en mutation, des acteurs face à de nouveaux défis. CIRAD/PRASAC Garoua, Cameroun, 27 au 31 mai 2002, $6 \mathrm{p}$.

Brévault T. 2010. Lutte contre les insectes ravageurs en culture Cotonnière : une agriculture moins dépendante des pesticides. $2 \mathrm{p}$

Bruinsma, W. 1987. Le Cotonnier In Entomologie Appliquée. AGRHYMET: Pays Bas ; 97.

Bruno M, Togola M, Téréta I, Traoré N. 2000. $\mathrm{La}$ lutte contre les ravageurs $\mathrm{du}$ cotonnier au Mali : problématique et évolution récente. Cahiers Agricultures, 9(2): 109-115.

Buès R, Boudinhon L. 2003. Écologie des invertébrés. Cahiers Agricultures, 12(3): 167-73.
Cadou J. 1982. Niveau de protection phytosanitaire et rendement en culture cotonnière pluviale au Mali. Cot. Fib. Trop., 37 : 317-325.

Cauquil J. 1993. Maladies et Ravageurs $d u$ Cotonnier en Afrique au Sud $d u$ Sahara. CIRAD : Montpellier, France $92 \mathrm{p}$.

CNUCED (Conférence des Nations Unies pour le Commerce et le Développement), 2003. Le développement économique en Afrique. UNCTAD/GDS/AFRICA /2003/1 $95 \mathrm{p}$.

Diongue I. 1989. Evolution récente du complexe des ravageurs du cotonnier au Sénégal et perspectives de la protection phytosanitaire. Revue Sénégalaise des Recherches Agricoles et Halieutiques, 2(2) : 31 - 41.

Enda diapol, 2009. Helicoverpa armigera, l'insecte ravageur le plus nuisible au monde. Daba, bulletin trimestriel d'informations sur l'agriculture et les biotechnologies $\mathrm{N}^{\circ}$ 03. Dakar: Enda diapol/Aproca, 7-8.

FAO. 2011. Produire Plus avec Moins. Guide à l'Intention des Décideurs sur l'Intensification Durable de l'Agriculture Paysanne. FAO: Rome, $106 \mathrm{p}$.

Ferron P, Deguine JP, Ekorong J. 2006. Évolution de la protection phytosanitaire du cotonnier : un cas d'école. Cahiers Agricultures, 15(1): $128-134$.

Guèye MT. 2011. Lutte contre les ravageurs des stocks de céréales et de légumineuses au Sénégal et en Afrique occidentale : une revue. BASE Biotechnol. Agron. Soc. Environ. 15(1): 183-194.

Héma SO. 2004. Contribution à la caractérisation biochimique de la résistance de Helicoverpa armigera (Hübner, 1808) (Lepidoptera, Noctuidae) au Burkina Faso. DEA. Ouagadougou : UO, 37p. 
Kébé B. 1999. Mise en Place d'un Système d'Alerte et d'Avertissement Agricole sur le Coton, Analyse d'un Suivi Phytosanitaire au Sénégal. Mémoire de fin de cycle, Ingénieur agronome. Thiès: E.N.S.A, 60 p.

Martin T. 2003. La résistance aux insecticides de Helicoverpa armigera (Hübner) en Afrique de l'Ouest : du mécanisme à la gestion. Thèse, Chimie. Toulouse : UT III, 77-[60] p.

Matthews GA, Tunstall JP. 1994. Insect Pests of Cotton. CAB International: Wallingford; 593p.

Mbaye DF. 2008. Interdiction de l'Endosulfan dans les pays du Sahel en Afrique de l'Ouest. Rapport de consultation, groupe de travail PAN/IPEN sur les pesticides POPs. Dakar: PAN AFRICA/IPEN, 55 p.

Oerke EC, Dehne HW. 2004. Safeguarding production - losses in major crops and the role of crop protection. Crop Protection, 23: 275-85.

Ouédraogo A, Yombi L, Doumbia S., Eyhorn F, Dischl R. 2008. Guide de production $\mathrm{du}$ coton biologique et équitable. Ouagadougou, Burkina Faso : Helvetas, 47p.

PR-PICA (Programme Régional de Protection Intégrée du Cotonnier en Afrique), 2013. Rapport synthèse des activités de recherche du Programme Régional de Protection Intégrée du Cotonnier en Afrique 2007-2012, 49p.

Prudent P, Loko S, Vaissayre M. 2006. Controlling an insecticide-resistant bollworm in West-Africa. Agriculture,
Ecosystems \& Environment, 107: 409411.

Smith CW, Cothren JT. 1999. Cotton: Origin, History, Technology, and Production. John Wiley \& Sons, Inc.: New York; 850 .

Tiemtoré CB. 2007. Rôle des plantes hôtes dans le maintien de la sensibilité de Helicoverpa armigera (Hübner,) (Lepidoptera : Noctuidae) aux toxines du coton $B t$ au Burkina Faso. DEA, Institut du Développement Rural, Université Polytechnique de Bobo, $43 \mathrm{p}$.

Traoré O. 2008 - Les succès de la lutte intégrée contre les ravageurs du cotonnier en Afrique de l'Ouest, 67ème réunion plénière de l'ICAC. Ouagadougou (Burkina Faso), 11p.

UEMOA. 2010. Coton de l'UEMOA : L'Or Blanc d'Afrique de l'Ouest à la Conquête du Marché Mondial. Ouagadougou, Burkina Faso: UEMOA, 21p.

Vaissayre, M. 1995. Ecological attributes of major cotton pests: implications for management. In Proceedings of the World Cotton Research Conference I, Challenging the Future, Constable GA, Forrester NW (eds). Brisbane, Australia, February 14-17 1994. Melbourne : Commonwealth Scientific and Industrial Research Organisation (CSIRO). 